\title{
A NOTE ON THE CONNECTEDNESS PROBLEM FOR NEST ALGEBRAS
}

\author{
DAVID R. PITTS
}

(Communicated by Paul S. Muhly)

\begin{abstract}
It has been conjectured that a certain operator $T$ belonging to the group $\mathscr{G}$ of invertible elements of the algebra $\operatorname{Alg} Z$ of doubly infinite uppertriangular bounded matrices lies outside the connected component of the identity in $\mathscr{G}$. In this note we show that $T$ actually lies inside the connected component of the identity of $\mathscr{G}$.
\end{abstract}

Let $\mathbf{T}$ be the unit circle in the complex plane with normalized Lebesgue measure. For $1 \leq p \leq \infty$, let $H^{p}$ be the usual Hardy space of all functions in $L^{p}(\mathbf{T})$ that have analytic extensions to the open unit disk $\mathbf{D}$. Let $\mathscr{H}=L^{2}(\mathbf{T})$ and let $\mathscr{B}(\mathscr{H})$ be set of all bounded linear operators on $\mathscr{H}$. Let $W \in \mathscr{B}(\mathscr{H})$ be the shift operator: $(W f)\left(e^{i \theta}\right)=e^{i \theta} f\left(e^{i \theta}\right)$. In this paper, we consider the nest $\left\{W^{n} H^{2}: n \in \mathbf{Z}\right\}$ of subspaces of $L^{2}(\mathbf{T})$, and its associated nest algebra,

$$
\operatorname{Alg} \mathbf{Z}=\left\{T \in \mathscr{B}(\mathscr{H}): T W^{n} H^{2} \subseteq W^{n} H^{2} \text { for all } n \in \mathbf{Z}\right\}
$$

A question which has been unanswered for several years is the following:

Question. Is the group of invertible elements of the Banach algebra $\mathrm{Alg} Z \mathbf{Z}$ connected in the norm topology?

It is frequently conjectured that the answer to this question is no. The reason for conjecturing a negative answer is because of a strong analogy between nest algebras and analytic function theory. We refer the reader to the book by Davidson [1] for details and more background on this question.

For each $f \in L^{\infty}(\mathbf{T})$, let $M_{f} \in \mathscr{B}(\mathscr{H})$ be the multiplication operator,

$$
M_{f} \phi=f \phi, \quad \phi \in L^{2}(\mathbf{T}) .
$$

Note that for $f \in H^{\infty}$, we have $M_{f} \in \operatorname{Alg} \mathbf{Z}$. Let $a$ be a positive real number and set

$$
h(z)=\frac{a i}{\pi} \log \left(\frac{1+z}{1-z}\right) .
$$

Received by the editors July 19, 1990.

1980 Mathematics Subject Classification (1985 Revision). Primary 47D25; Secondary 46B35.

Key words and phrases. Nest, nest algebra.

Research partially supported by NSF grant DMS-8702982 and by an NSF Mathematical Sciences Postdoctoral Fellowship. 
Then $h$ is a conformal map of the open unit disk onto the unbounded vertical strip $\{z \in \mathbf{C}:-a<\operatorname{Re}(z)<a\}$.

If $f=\exp (h)$ then it is easy to see that both $f$ and $1 / f$ are $H^{\infty}$ functions and moreover, that $f$ is not the exponential of any $H^{\infty}$ function. Therefore $f$ cannot be connected to the constant function 1 via a norm continuous path within the group of invertible elements of the Banach algebra $H^{\infty}$. For this reason, the operator $M_{f}$ has been suggested as a possible example of an operator which cannot be connected to the identity via a norm continuous path inside the group of invertibles in $\operatorname{Alg} \mathbf{Z}$.

The purpose of this note is to show that in fact, $M_{f}$ may be connected to the identity via a norm continuous path of invertible elements in $\operatorname{Alg} \mathbf{Z}$.

Before giving the proof we pause for some terminology and to make a few simple remarks.

Let $\mathscr{A}$ be a unital Banach algebra with unit $I$. Say that an invertible element $a$ of $\mathscr{A}$ may be connected to the identity if thee exists a norm continuous function $f:[0,1] \rightarrow \mathscr{A}$ such that $f(0)=a, f(1)=I$, and $f(t)$ is an invertible element of $\mathscr{A}$ for each $t$. The algebra $\mathscr{A}$ has the connectedness property if every invertible element of $\mathscr{A}$ may be connected to the identity. We use the term symmetry to describe a square root of the identity in a unital Banach algebra $\mathscr{A}$. Such elements have spectrum contained in the set $\{-1,1\}$ and hence are connected to the identity. In fact, if $\gamma(t)$ is an arc in the complex plane connecting -1 to 1 which does not pass through the origin, then

$$
\sigma(t)=\frac{I+S}{2}+\gamma(t) \frac{I-S}{2}
$$

is a norm continuous path of invertible elements of $\mathscr{A}$ which connects the symmetry $S$ to the identity $I$.

The algebra

$$
\mathscr{D}=\operatorname{Alg} \mathbf{Z} \cap(\operatorname{Alg} \mathbf{Z})^{*}
$$

is a von Neumann subalgebra of $\operatorname{Alg} \mathbf{Z}$ and since any von Neumann algebra has the connectedness property, we see that any invertible operator in $\mathscr{D}$ can be connected to the identity in $\operatorname{Alg} Z$.

Remark. Let $\alpha$ be a complex number of unit modulus and let $g \in L^{\infty}(\mathbf{T})$. Let

$$
g_{\alpha}(z)=g(\alpha z), \quad z \in \mathbf{T},
$$

and define a unitary operator $S_{\alpha} \in \mathscr{D}$ by

$$
S_{\alpha} e_{n}=\alpha^{n} e_{n},
$$

where $e_{n}\left(e^{i \theta}\right)=e^{i n \theta}$ is the usual orthonormal basis for $L^{2}(\mathbf{T})$.

We then have

$$
S_{\alpha} M_{g} S_{\alpha}^{*}=M_{g_{\alpha}} .
$$

Note that by the above remarks, $M_{g}$ and $M_{g_{\alpha}}$ belong to the same connectedness class of invertibles in $\mathrm{Alg} \mathbf{Z}$.

We now show that $M_{f}$ can be connected to the identity. Note that $h(z)=$ $-h(-z)$. It follows that we have

$$
f(z) f(-z)=1 \quad \text { for all } z \in \overline{\mathbf{D}} .
$$


If $S=S_{-1}$, equation (2) yields,

$$
S M_{f} S M_{f}=I .
$$

Hence both $S$ and $S M_{f}$ are symmetries in $\mathrm{Alg} \mathbf{Z}$ and

$$
M_{f}=S\left(S M_{f}\right) \text {. }
$$

Therefore $M_{f}$ can be connected to the identity in $\operatorname{Alg} \mathbf{Z}$. Moreover, equation (1) enables one to obtain an explicit path connecting $M_{f}$ to the identity.

Question. Let $m$ be a conformal mapping of the disk onto itself and set $g=$ $f \circ m$. Is $M_{g}$ connected to the identity in $\operatorname{Alg} \mathbf{Z}$ ? Note that the remark above shows that if $m$ is a rotation, then this is the case.

Remark. Let $R$ be any proper open subset of the complex plane that is simply connected and satisfies $R=-R$. Then $0 \in R$ and if $h$ is any conformal map from the disk onto $R$ with $h(0)=0$, we have $h(z)=-h(-z)$. (Indeed, the function $g(z)=-h(-z)$ is also a conformal map of the disk onto $R$. Since $h(0)=g(0)$ and $h^{\prime}(0)=g^{\prime}(0)$, the Riemann mapping theorem implies $g=h$.) The argument given above now shows that if we assume that $\{\operatorname{Re}(z): z \in R\}$ is bounded and set $f=\exp (h)$, then $M_{f}$ is a product of two symmetries in $\operatorname{Alg} \mathbf{Z}$ and hence is connected to the identity in $\operatorname{Alg} \mathbf{Z}$.

\section{REFERENCES}

1. K. R. Davidson, Nest Algebras, Research Notes in Math., vol. 191, Pitman, Boston, London, and Melbourne, 1988.

2. Z. Nehari, Conformal mapping, 1st ed., Internat. Ser. Pure and Appl. Math., McGraw-Hill, New York, Toronto, and London, 1952.

Department of Mathematics and Statistics, University of Nebraska, Lincoln, NeBRASKA, 68588 90024

Current address: Department of Mathematics, University of California, Los Angeles, California 